Action and word learning

\title{
Parallels between action-object mapping and word-object mapping in young children
}

\author{
Kevin J Riggs \& Emily Mather \\ Department of Psychology, University of Hull, Hull, HU6 7RX, UK \\ Grace Hyde \& Andrew Simpson \\ Department of Psychology, University of Essex, Wivenhoe Park, Colchester, Essex, \\ CO4 3SQ, UK
}

Corresponding author: Dr Kevin Riggs, Department of Psychology, University of Hull, Cottingham Road, Hull, HU6 7RX, UK

Email: k.riggs@hull.ac.uk

Keywords: fast mapping, actions, language, word learning

This is the peer reviewed version of the following article: Riggs, K. J., Mather, E., Hyde, G. and Simpson, A. (2016), Parallels Between Action-Object Mapping and Word-Object Mapping in Young Children. Cognitive Science, 40: 992-1006. doi: 10.1111/cogs.12262, which has been published in final form at http://onlinelibrary.wiley.com/doi/10.1111/cogs.12262/abstract. This article may be used for non-commercial purposes in accordance With Wiley Terms and Conditions for self-archiving. 
Action and word learning

\begin{abstract}
Across a series of four experiments with 3- to 4-year-olds we demonstrate how cognitive mechanisms supporting noun learning extend to the mapping of actions to objects. In Experiment $1(n=61)$ the demonstration of a novel action led children to select a novel, rather than a familiar object. In Experiment $2(n=78)$ children exhibited long-term retention of novel action-object mappings, and extended these actions to other category members. In Experiment $3(n=60)$ we showed that children formed an accurate sensorimotor record of the novel action. In Experiment $4(n=54)$ we demonstrate limits on the types of actions mapped to novel objects. Overall these data suggest that certain aspects of noun mapping share common processing with action mapping and support a domain-general account of word learning.
\end{abstract}


Children are prodigious word learners. By the age of seventeen, the average Englishspeaker knows more than 60,000 words (Bloom, 2000). The acquisition of nouns in particular starts from an early age. Even before children reach their first birthday, they have already begun learning the names of commonplace objects (Bergelson \& Swingley, 2013). How children make light of such a complex inductive task has been explained in a variety of ways: domain-specific biases (e.g., Golinkoff, Mervis, \& Hirsh-Pasek, 1994; Waxman and Booth, 2000); social pragmatism (e.g., Bloom, 2000; Akhtar, Carpenter, \& Tomasello, 1996); and domain-general aspects of learning, memory, and attention (e.g., Samuelson \& Smith, 1998; Smith \& Yu, 2008). Critically, the domain-specific perspective implies that the powerful mental processes used to map names to objects are exclusive to the domain of language. In this paper, we investigate whether these processes extend to learning in other domains.

The computational challenge of the task (Quine, 1960), combined with rapid vocabulary growth in early childhood, suggests a domain-specific solution (Golinkoff et al., 1994). However, young children are also sophisticated perceptual and statistical learners (e.g., Saffran, Aslin \& Newport, 1996; Kirkham, Slemmer, \& Johnson, 2002). Experimental and computational research demonstrates that general learning mechanisms may suffice for certain aspects of word learning (see e.g., Samuelson, 2002; Smith, Jones, Landau, GershkoffStowe, \& Samuelson, 2002). Different approaches have been taken to investigate the generality of word learning processes. One method has been to explore how general processing mechanisms, such as statistical learning or attention, operate in word learning (e.g., Smith \& Yu, 2008; Smith et al., 2002). A different approach, and the one adopted in the present research, is to determine whether the processes used to map words to objects extend to mapping other kinds of information to objects (e.g., Childers \& Tomasello, 2003). Specifically, we investigate the extent to which various aspects of word mapping extend to action mapping. 
The task of word learning decomposes into different processes which unfold over different timescales. For example, a distinction can be made between the initial identification of the correct referent for a noun and the longer-term retention of the mapping (see e.g., Horst \& Samuelson, 2008; Swingley, 2010). The domain-generality of each process needs to be considered. The initial disambiguation process has perhaps been the most extensively researched aspect of word learning. For example, when shown a novel and a familiar object, and upon hearing a new word, children map the new word to the novel object. We term this behaviour 'novel referent selection'. Various explanations have been proposed to account for this behaviour. Some of these explanations invoke a specifically linguistic constraint, such as the mutual exclusivity constraint (e.g., Markman, 1990), the novel name-nameless category (N3C) principle (Mervis \& Bertrand, 1994; Golinkoff, Mervis, \& Hirsh-Pasek, 1994) and the principle of contrast (Clark, 1987). Alternatively, novel referent selection could be the outcome of a broader learning bias, such as attention to novelty (Mather \& Plunkett, 2012; Horst, Samuelson, Kucker \& Murray, 2011), or general social-pragmatic reasoning (Grassmann, Stracke \& Tomasello, 2009). While no studies to date have investigated novel referent selection in relation to action-object mapping, a few studies have shown that children map novel verbs to novel actions (Merriman, Marazita, \& Jarvis, 1995; Merriman, EveyBurkey \& Jarvis, 1996). These demonstrations are nonetheless restricted to the mapping of linguistic information.

Beyond disambiguation, or novel referent selection, children also need to retain word mappings over time and extend labels to other category members. To this end, researchers have investigated how well children remember new words. A classic study by Carey and Bartlett (1978) reported that preschool children rapidly learn new words under conditions of minimal exposure (e.g., one or two presentations) and retained these mappings a week later. 
Markson and Bloom (1997) not only investigated whether word-object mappings are retained over time, they also investigated if other information is mapped. Three- to four-yearold children were introduced to novel objects in three different conditions: an object label condition ("let's use the $k o b a$ "); a linguistically-presented fact condition ("My uncle gave me this"); and a visually-presented fact condition (a sticker placed on a particular object). At test, they were shown the same novel objects and asked to "Find the koba", "Find the one my uncle gave me", or "Put the sticker on its object". When tested immediately, children and adults performed well on all three conditions. Long-term retention was measured one week and one month later. Both children and adults remembered the object associated with the label and the linguistically-presented fact - however, performance was significantly worse in the visually-presented fact condition than the other two conditions with children selecting the correct object no better than chance. Markson and Bloom (1997) interpreted similar performance in the linguistic-fact and object-label conditions as support for the view that word learning does not rely on specifically linguistic processes.

More recently, Childers and Tomasello (2002) investigated whether the impressive retention of word mappings extends to action mappings. They trained young children on novel nouns, verbs and actions associated with a novel object (the actions employed to use the object). They tested comprehension ("Which object can I do this with?" as the experimenter performed the novel action) at time intervals of one minute, one day, and one week. Memory for correct actions was very good for all time intervals. However, children in this study received at least four exposures to the new action, and many received eight exposures, over a month of training. Thus, the authors did not provide evidence that novel actions are remembered for a substantial period of time as a result of minimal exposure - as is the case with word learning in the studies by Carey and Bartlett (1978) and Markson and Bloom (1997). 
In a follow up study, Childers and Tomasello (2003) further investigated novel word and action learning. They also sought evidence that children extend action mappings to other category members, as is the case when children learn new words. This time children were trained over only a 15-minute period with several objects, actions, and words, and tested immediately after exposure. At testing the researchers again assessed comprehension. Performance with actions was good, and no different from novel word comprehension. However, although the training phase was considerably shorter than in their earlier paper, testing only took place immediately after training. A similar issue applies to more recent data on word and action production reported by Hahn and Gershkoff-Stowe (2010). In this study 2- and 3-year-old children watched the experimenter demonstrate four novel actions with four novel objects. Straight afterwards, children were asked which action they would perform with each of the four objects. Action production was good, and better than word production. Thus, although there is evidence for good action-object mapping immediately after exposure, we currently have no evidence that the action associated with an object's use, learnt under minimal exposure conditions is retained long term, and extended to other category members.

In the research reported here we sought further evidence for commonalities between word-object mapping and action-object mapping. In Experiment 1 we undertook an investigation into whether young children use a novel action to select a novel referent. Children were told that the action demonstrated by the experimenter was the action related to the object's use. In Experiment 2, we asked whether children retain rapidly-learnt actions in the long term, and extend these action mappings to other category exemplars. Learning took place under minimal exposure conditions with the action being performed only twice. In Experiment 3 we investigated the fidelity of the learnt action representation, and in Experiment 4 we sought to establish whether it is only those actions associated with an object's use that are readily learnt and retained long term. 


\section{Experiment 1}

\section{Participants}

Sixty-one children took part in the experiment (31 girls, 30 boys). The children were aged between 3 years, 1 month and 4 years, 3 months (mean age 3 years, 8 months) and attended nursery schools in a semi-rural county of England. All spoke English as a first language and the class teacher reported that none had behavioral or educational problems. The majority of children were from a white and middle-class background.

\section{Materials}

Four familiar and four novel objects were used (see Fig. 1). The four familiar objects were a cup, a small hairbrush, children's plastic scissors and a toy hammer. The four novel objects were a four-way radiator key, a leaf guard, a plastic hoof-pick and a hose-part. The same familiar and novel objects were paired giving pairings of cup/leaf-guard, brush/radiatorkey, scissors/hoof-pick and hammer/hose-part. Teacher report confirmed that the children had experience of using the familiar objects (e.g., drinking from cups at milk time). They also confirmed that the novel objects were unlikely to have been seen or used by the children in class. This use of teacher report was also employed in all subsequent experiments.

Figure 1 about here

\section{Procedure}

In a mixed-groups design, children were randomly assigned to either an Action or a Word condition, with two of the four object pairs, and received either the familiar or novel name/action first (so that object pair and order-of-familiarity were counterbalanced between 
children). Children in the Action condition were tested with both a familiar and novel action, while children in the Word condition were tested with both a familiar and novel word. Thus the independent variables were Information type (Word, Action), Action/Name familiarity (Familiar, Novel), Object pair (cup/leaf guard, brush/radiator key, scissors/hoof-pick, hammer/hose-part) and Order (Familiar-first, Novel-first). The dependent variable was frequency of selection of the familiar object.

The experimenter (E) and the child sat at a table with a piece of paper, some crayons and a pair of bags (one large and one small). Each bag contained two objects (one familiar the other novel). Children were invited to play a drawing game with E, where they were asked to draw around their own hand, after which they were asked to draw around E's hand. The paper was then turned over and the procedure differed according to condition. In the Word condition (Novel-first), E asked the child "Look in the big bag. Can you give me the Koba for us to draw around?" Children chose one of the two objects from the big bag and then drew around it. Then E asked the child "Look in the small bag. Can you give me the $\mathrm{X}$ for us to draw around?" (X= cup, brush, scissors or hammer). Children again drew round it. In the Action condition (Novel-first), children were asked "Look in the big bag. Can you give me the thing we do this with for us to draw around?" (while E demonstrated the action of rubbing the right hand against the upper left arm). Then E asked the child "Look in the small bag. Can you give me the thing we do this with for us to draw around?" (while E demonstrated the action for the familiar object in the bag - for example, making a hair brushing action).

\section{Results and Discussion}

The frequency of selecting the familiar object differed according to the familiarity of the word or action. The majority of children chose the familiar object when presented with a familiar word (26/30) or a familiar action (25/31). In contrast, when presented with a novel 
word $(7 / 30)$ or action $(8 / 31)$ the majority of children did not choose the familiar object.

Children selected the familiar object more often than predicted by chance $(p=.5)$, when a familiar word or action was presented (binomial, $p<0.001$ ); and less often with a novel word or action (binomial, $p<0.001$ ).

Chi-squared analyses did not reveal any significant differences in the frequency of selecting the familiar object in response to a familiar word compared to a familiar action $\left(X^{2}(1)=.403, p=.52\right)$, or an unfamiliar word compared to an unfamiliar action $\left(X^{2}(1)=.050\right.$, $p=.82)$. Neither were there object pair effects: that is, the frequency of selecting the familiar object did not differ across the four object pairs (cup/leaf guard, brush/radiator key, scissors/hoof-pick or hammer/hose part). Data were analyzed using Fisher's exact test because some cells had counts less than five. For familiar words and actions, $p=.166$, and for unfamiliar words and actions, $p=.605$.

Similar to the referent selection data from word learning studies, children exclude as potential referents for novel actions, any object whose use they are familiar with. Our findings suggest that children will reject additional actions for familiar objects which they already know how to use. In turn, this suggests that the exclusion learning seen when children hear new words extends to observing novel actions. What might drive behavior to exclude the familiar object? Recent data from word learning research suggests that maybe children's attention is biased towards the most novel stimulus in a referent selection task (Mather \& Plunkett, 2012; Horst, Samuelson, Kucker \& McMurray, 2011; see also Merriman \& Bowman, 1989), or even a bias away from the familiar (McMurray, Horst \& Samuelson, 2013). Future work needs to address the specific role that novelty might play in how novel actions are used to select novel objects.

\section{Experiment 2}

We established in Experiment 1 that young children will use novel action information 
to infer (select) a novel referent. In Experiment 2 children were not asked to infer the actionobject mapping, but were instead given the mapping directly. Here our focus was whether children would retain novel action-object mappings long-term, and extend these actions to exemplars from the same category. Showing that novel actions do extend to new exemplars (like new words) would provide further evidence that there are cognitive parallels between action and word learning. In the learning session we ensured that the novel action was presented under minimal exposure conditions (i.e., performed only twice) similar to those used in word learning research. We also ensured that the action did not produce an observable outcome - the novel object was rubbed on the upper part of the left arm without any obvious effect (as in Childers \& Tomasello, 2003). This made it highly unlikely that children could have inferred the action by remembering its effect (e.g., inferring a hammering action by remembering that it caused breaking). With an "effectless" action we could be confident that children were learning the observed action, not merely recreating it from the observed outcome when asked at testing.

\section{Method}

\section{Participants}

Seventy-eight children, who had not previously been tested, took part in the experiment (38 girls, 40 boys). The children were aged between 3 years, 3 months and 4 years, 3 months (mean age 3 years, 11 months) and attended two nursery schools approximately two kilometres apart in outer London, England. They spoke English as a first language and the class teacher reported that none had behavioral or educational problems.

\section{Materials}

In the exposure session children were shown five objects. Following the word learning procedure of Bloom and Markson (1997) we used a mix of familiar and novel objects. Two objects were familiar (a green felt pen and a red pencil sharpener). There were three novel 
objects (a white T shaped plastic plumbing fitting, an orange $\mathrm{L}$ shaped plastic bracket and a black staple remover). None of the novel objects afforded (or suggested) the novel action (arm rubbing) and each one served as the target object on 1/3rd of trials. In the testing sessions five new objects were introduced that were the same shape as the original learning objects but differed in both colour and size. The familiar objects were this time an orange felt pen and a green pencil sharpener. The novel objects were a smaller black plumbing fitting, a larger white L shaped bracket, and a larger red staple remover.

\section{Procedure}

Children were randomly assigned to either a Novel-action or a Novel-word condition. The independent variable was novel Information type (Word, Action). The dependent variable was selection accuracy.

\section{Exposure session}

Participants were tested individually in a quiet corridor of the school and were asked to play a fun "game" with the experimenter (E) sitting opposite the child at a table. Initially the procedure was identical in both conditions. E placed a sheet of paper (300mm x $420 \mathrm{~mm}$ ) containing outline drawings of the five test objects on the table. E then showed the child a brown bag and emptied the 5 test objects onto the table asking the child to play a game of matching up the objects with the line drawings on the paper. Most children found this very easy but were helped with the matching if needed. Upon completion of the matching, E then told the child that they were going to put the objects away, one-by-one, into the bags that $\mathrm{E}$ had brought along (a red bag and a blue bag). Children were allowed to choose which bag (red or blue) each object went into. E then pointed first to one of the novel objects and instructed the child to hand it across while asking them which bag ("this thing") should go into. Children chose either the red or the blue bag and E placed the object inside. This was repeated for the four remaining objects in the following order - familiar object, novel object 
(the target object in both conditions), familiar object, novel object. At the point the child handed over the target object (the third object) the procedure differed according to condition.

In the Novel-word condition, E pointed at the novel target object and asked the child to hand across the 'koba'(all children handed over the target object). When E received the object the child was asked which bag the 'koba' should go into. Once the child had responded the object was placed inside the chosen bag. Thus the novel word was mentioned only twice.

In the Novel-action condition, E pointed at the novel target object and asked the child to hand across the object "we do this with" (while performing the action of rubbing her right hand up and down her left upper arm). All children handed over the target object. When E received the object the child was asked which bag the object "we do this with" (performing again the action of rubbing her right hand up and down her left upper arm) should go into. Once the child had responded the object was placed inside the chosen bag. Thus the novel action was performed only twice - once without the object in hand (when E asked for it to be handed across) and once with it in hand (before the object was placed in the red/blue bag).

\section{Delayed test session}

After five to seven days the experimenter returned and asked the child to play the matching game again (where the objects were matched with their line drawing equivalents). The test objects differed from the objects in the exposure session because although they were the same shape, they differed in both size and colour. After the objects were placed on the paper, children were asked "Can you point to the "koba?" in the Novel-word condition and "Can you point to the thing we do this with?" (while performing the action of rubbing the upper arm) in the Novel-action condition. Children were then thanked for their help in the game and shown back to their class.

\section{Results and Discussion}


At testing, five to seven days later, the majority of children chose the correct object in both the Novel-word (26/39) and the Novel-action (24/39) conditions. In the Novel-action condition correct responses were evenly distributed across the three novel objects. Overall, children performed significantly above chance performance of 1/5 (binomial, $p<.001$ ) assuming that children regarded both familiar and novel objects as potential referents; and 1/3 (binomial, $p<.001$ ) assuming that children only regarded novel objects as potential referents. As in Experiment 1, the pattern of responding was similar across the word and action conditions with no significant difference in accuracy (odds ratio $=1.25, p=.64$ ).

Our data suggest that under minimal learning conditions (two exposures to the new word or action), children can remember the novel action associated with the novel object long-term (in this case approximately one week). Moreover, children did not merely form a long term representation between a novel action and a particular novel object - children extended this mapping to other object exemplars from the same category in the same way as they do for words (Waxman \& Booth, 2000; Smith, Jones, Landau, Gershkoff-Stowe \& Samuelson, 2002). Thus we demonstrate for the first time, under exposure conditions very similar to those encountered in word learning that children readily learn, and retain with extension, the novel actions associated with novel objects.

\section{Experiment 3}

As an extension of Experiment 2, we deemed it necessary to determine both the specificity of the action mapping and indeed whether a mapping had been formed at all. It is possible that children directed their test responses towards whichever object was most similar to the object 'singled out' during the exposure phase. Such a response tendency does not require the child to have mapped the action to the object. Furthermore, even if the action was mapped, the children may have only remembered that a 'strange' action was associated with the object. 
We reasoned that if children merely remembered that a 'strange' action was associated with the target object, or had not formed a mapping at all, then they would select the target object at test even when a different novel action was modelled. If, on the other hand, they had mapped a detailed representation of the first novel action to the target object, then they would not select this object when presented with a new novel action. Instead, children would select one of the other novel objects presented in the testing session. Therefore, we sought to replicate the novel-action condition of Experiment 2, and contrast it with a condition in which children were presented with a new novel action at test. Hence, one novel action was performed with the target object in the exposure session, but a different novel action was performed in the testing session.

\section{Method}

\section{Participants}

Sixty children, who had not previously been tested, took part in the experiment (29 girls, 31 boys). They were aged between 3 years, 2 months and 4 years, 4 months (mean age 3 years, 10 months). All attended a nursery in a middle class borough of London,

England. They spoke English as a first language and the class teacher reported that none had behavioral or educational problems.

\section{Procedure}

Half the children were randomly assigned to a Novel-action condition (as used in Experiment 2). The other half were assigned to the new Second-novel-action condition. The exposure session was identical to that reported in the Novel-action condition of Experiment 2 (the upper left arm was rubbed by the right hand).

In the testing session the two familiar and three novel objects were placed on the table between the child and the Experimenter, and the procedure differed according to condition. In the Novel-action condition children were asked to point to the object "we do this with" (while E performed the same action used at exposure). In the Second-novel-action 
condition children were asked to point to the object "we do this with" while E performed a second - hitherto unseen - action (E performed a circular motion on the table). Thus this second test action differed from the first "exposure" action both in terms of how the action was performed (linear motion/circular motion) and the substrate upon which the action was performed (the body/the table).

\section{Results and Discussion}

In the Novel-action condition performance was similar to that reported in Experiment 2. The majority of children (24/30) selected the correct object and their performance was above chance (binomial, $p<.001$ ), where chance was taken to be the more conservative one out of three novel objects. In the Second-novel-action condition, performance was poor with few children (5/30) selecting the target object modelled in the exposure session. Children actually selected it significantly less than predicted by chance (binomial, $p=.02$ ). Nearly all the remaining children selected one of the other novel objects (23/30), although two children selected one of the familiar objects.

These results further suggest that the children in Experiment 2 retained and extended a novel action mapping, rather than displaying a general preference for the target object at test. Furthermore, children are not merely remembering that a 'strange' action was performed on the target object. Had this been the case, then we would have expected many more children to have selected the target object at test in the second-novel-action condition. Rather, these results suggest that children retain an accurate representation of the novel action, which has been associated with the target object. They also provide robust support for the findings from Experiment 1. Twenty-three out of 30 children in the Second-novel-action condition selected one of the other two novel objects when presented with the second novel action during testing. These data support the view that children exclude as potential referents, objects for which they already have an associated action. In the final experiment we sought 
further evidence that children map novel actions to novel objects, specifically those actions related to an object's use.

\section{Experiment 4}

In their visually-presented fact condition, Markson \& Bloom (1997) report that children fail to retain information relating to attaching a sticker to a novel object. That is, children failed to map and retain information about a specific action performed on an object. In Experiments 2 and 3, we found that children readily map and retain (with extension) the actions associated with an objects use, i.e., those actions that are made with an object. In the final experiment we made a direct comparison between these two types of actions. In a Sticker condition, children were introduced to a novel object onto which the experimenter attached a small sticker. In an Action condition, the experimenter performed a novel userelated action with the novel object as before. If children remember any action performed in the vicinity of the novel object then we should expect good performance in both conditions and no difference between them. If, on the other hand, children readily learn only those actions related to how the object is used, then we should expect to see good performance in the Action condition only.

\section{Method}

\section{Participants}

Fifty-two children, who had not previously been tested, took part in the experiment (27 girls, 25 boys). They were aged between 3 years, 4 months and 4 years, 5 months (mean age 4 years, 0 months). All attended a nursery in a middle-class borough of London, England. They spoke English as a first language and the class teacher reported that none had behavioral or educational problems.

\section{Procedure}


Half the children were randomly assigned to an Action condition. The other half were assigned to the Sticker condition.

In both conditions children sat opposite the Experimenter $(\mathrm{E})$ at a table onto which were placed five novel objects. The child and E played a game of drawing around each of the novel objects on a piece of paper. E then asked the child to help tidy up and put the objects away. Pointing to one of the objects, E asked the child hand over the object so it could be put away in her bag. While the third object was being handed over (the same object in both conditions) the procedure differed according to condition. In the Action condition, E said "See this object, this is what we do with it" (the upper left arm was rubbed by the right hand) before placing the object in the bag. In the Sticker condition, E said "See this object, I'm going to put this sticker on it" (E stuck a small yellow sticker on it with her right hand) before placing the object in the bag.

In the testing session three days later, the procedure differed according to condition. In the Action condition, children were asked to point to the object "we do this with" (while performing the action of rubbing her upper left arm). In the Sticker condition, children were asked to point to the object "we put the sticker on last time"

\section{Results and Discussion}

Children recognised the target object from the exposure session more in the Action condition (18/26) than the Sticker condition (6/26). This difference was significant (odds ratio $=7.88, p=.001$ ); children's performance was above chance for the Action condition (binomial, $p<.001$ ) but not the Sticker condition (binomial, $p=.91$ ). These results suggest that while children learn how an object is used as readily as they learn what it is called, this learning does not extend to all actions performed in relation to that object. Two reviewers did however raise two further interpretations. Perhaps the action 'put a sticker on' is not a novel action for children: it is the familiar action associated with a sticker. Children may therefore 
have been reluctant to apply this familiar action to a novel object (rather like they are reluctant to apply a familiar label to a novel object). Another possibility is that the sticker (a familiar object) may have been so salient for children so as draw their attention away from the novel object thus hindering their action learning in the 'sticker' condition (see Simpson, Carroll \& Riggs, 2014, for a similar discussion about how the introduction of a familiar object may draw attention away from a novel one). Future work should investigate these alternative interpretations.

\section{General Discussion}

We aimed to establish whether children rapidly learn and retain information about how a novel object is used, in much the same way as they learn and retain information about what the novel object is called. Our data suggest that children learn and retain novel actions under exposure conditions similar to those encountered when they learn and retain new words. They use novel action information to select a novel referent, suggesting that the 'mutual exclusivity' response seen in word learning may be part of a more general exclusion response, or perhaps the result of a general bias to attend to novelty. They also retain novel actions long term, extending them to other objects from the same category. However, it is not the case that any novel action will be mapped to a novel object - we found that children do not remember to which novel object a sticker was attached. Our experiments support, consolidate and extend the findings of Childers and Tomasello $(2002,2003)$ and Hahn and GershkoffStowe (2010) that young children learn novel actions at least as readily as novel nouns.

Our findings support the view that word learning does not necessarily rely on specifically linguistic processes (Bloom, 2000). We take our data to suggest that the rapid learning and retention we see in children's word learning utilises more general processing extending, at least, to certain kinds of novel actions. Furthermore, our data support the view that the cognitive processes underlying referent selection and category extension are not 
restricted to the domain of word learning (see e.g., Mather \& Plunkett, 2012; Mayor \& Plunkett, 2010). Yet, it is not the case that any kind of information can be mapped to a novel object, as evidenced by the sticker condition of Experiment 4. What limits the scope of mapping, if it is not restricted to words alone? One possibility is that category relevant information is mapped. By category relevant information we mean information that is indicative of category membership, such as the category label or the action performed when using that kind of object (Bloom, 2000). It is more cognitively efficient to retain information shared across category members, rather than details about each specific member. Indeed, we found that novel words and actions were also extended to other category members. However, one potential problem for this view is that Markson and Bloom (1997) report the fast-mapping of the linguistic fact 'My uncle gave me this' - a linguistic fact that was unlikely to be category relevant.

Possibly, one might wish to suggest that two processing mechanisms are at work - one for word learning and one for action learning. However, such a suggestion is an unparsimonious way to explain our findings. In our data, we see similarities both in referent selection (Experiment 1) and in retention with extension (Experiments 2) for both words and actions. Moreover, this pattern of responding was observed under minimal exposure conditions. We suggest that such strong similarities are indicative of common processing underpinning both word and action learning. Nonetheless, we also note that the present research is limited to a particular age group, and it might be fruitful to look at younger children (e.g., 2-year-olds) in future research to establish the extent to which word mapping and action mapping exhibit similar developmental trajectories.

An exciting question for future work is whether the rapid learning and retention for words and actions share common neural processing. While nobody has yet addressed this question with regard to word learning, recent findings do suggest common brain functioning 
for symbolic manual gestures and spoken language $(\mathrm{Xu}$, Gannon, Emmorey, Smith \& Braun, 2009). It should be noted however that in our studies the actions were not symbolic gestures (a form of signed label), but the actions typically performed to use the objects (e.g., in Experiment 3 the experimenter said "...this is what we do with it"). A number of studies have looked at children's understanding of symbolic gestures in relation to novel objects, but whether children rapidly learn and retain these novel gestures to novel objects is unclear (e.g., see Suanda and Namy, 2013). Future work should seek to establish if children rapidly learn (and retain) signed labels in much the same way as they do new words and actions related to use.

Finally, it is important to reiterate that in our study the actions performed were essentially "effectless" actions. That is, the action occurred and no observable outcome was produced (e.g., the novel object was rubbed up and down the arm). That children nonetheless attended to these actions is entirely consistent with findings from social learning research where a growing body of data suggests that children are heavily focussed on actions in social learning tasks, even when these actions seem irrelevant to any outcome - often termed the problem of overimitation (Whiten, McGuigan, Marshall-Pescini \& Hopper, 2009). Interestingly, comparative research tells us that non-human primates are much less interested in actions than they are in outcomes, and there is no evidence that they overimitate (e.g., Horner \& Whiten, 2005). Given that non-human primates do not learn language it would be interesting to see if they were also unable to map novel actions to novel objects. Such an observation would further support the view that action-object mapping and word-object mapping share common processing.

In summary, children rapidly learn and retain the novel actions associated with novel objects in much the same way they rapidly learn the novel names of novel objects. Rapid learning, coupled with long term retention and extension, is not limited to word learning. 
Action and word learning

Moreover, this ability to rapidly learn actions, actions that do not produce any observable outcome, may be a uniquely human ability and future work should seek to investigate more fully the relation between action and word learning. 


\section{References}

Akhtar, N., Carpenter, M. \& Tomasello, M. (1996). The role of discourse novelty in early word learning. Child Development, 67, 635-645.

Bloom, P. \& Markson, L. (1998). Capacities underlying word learning. Trends in Cognitive Science, 2, 67-73

Bloom, P. (2000). How Children Learn the Meaning of Words. Cambridge, MA: MIT Press

Carey, S. \& Bartlett, E. (1978). Acquiring a single new word. Papers and Reports on Child Language Development, 15, 17-29

Childers, J.B. \& Tomasello, M. (2002). Two-year olds learn novel nouns, verbs, and conventional actions from massed or distributed exposures. Developmental Psychology, 38, 967-78.

Childers, J.B. \& Tomasello, M. (2003). Children extend both words and non-verbal actions to novel exemplars. Developmental Science, 6, 185-90

Clark, E. (1987). The principle of contrast: A constraint on language acquisition. In B. MacWhinney, (Ed.) Mechanisms of language acquisition (pp 1-33). Hillsdale, NJ: Lawrence Erlbaum Associates.

Gershkoff-Stowe, L. \& Hahn, E. (2013). Word comprehension and production asymmetries in children and adults. Journal of Experimental Child Psychology, 114, 489-509

Golinkoff, R. M., Mervis, C. B. \& Hirsh-Pasek, K. (1994). Early object labels - the case for a developmental lexical principles framework. Journal of Child Language, 21(1), 125155 .

Grassmann, S., Stracke, M. \& Tomasello, M. (2009). Two-year-olds exclude novel objects as potential referents of novel words based on pragmatics. Cognition. 112, 488-493

Hahn, E. \& Gershkoff-Stowe, L. (2010). Children and adults learn actions for objects more readily than labels. Language Learning and Development, 6, 283-308

Horner, V. \& Whiten, A. (2005). Causal knowledge and imitation/emulation switching in chimpanzees and children. Animal Cognition, 8, 164-181.

Horst, J. S. \& Samuelson, L. K. (2008). Fast mapping but poor retention by 24-month-old infants. Infancy, 13(2), $128-157$.

Horst, J. S., Samuelson, L. K., Kucker, S. C. \& McMurray, B. A. (2011). What's new? Children prefer novelty in referent selection. Cognition, 118 (2), 234-244

Kirkham, N. Z., Slemmer, J. A. \& Johnson, S. P. (2002). Visual statistical learning in infancy: Evidence for a domain general learning mechanism. Cognition, 83, B35-B42. 
Markman, E. M. (1989). Categorization and naming in children: Problems of induction. Cambridge, MA: MIT Press.

McMurray, B.A, Horst, J.S. \& Samuelson L.K. (2012). Word learning emerges from the interaction of online referent selection and slow associative learning. Psychological Review, 119(4), 831-877

Markman, E. M. (1990). Constraints children place on word meanings. Cognitive Science, 14(1), 57-77.

Markson, L. \& Bloom, P. (1997). Evidence against a dedicated system for word learning in children. Nature, 385, 813-815.

Merriman, W. E. \& Bowman, L. L. (1989). The mutual exclusivity bias in children's word learning. Monographs of the Society for Research in Child Development, 54(3-4), Serial Number No. 220.

Merriman, W. E., Evey-Burkey, J. A., Marazita, J. M. \& Jarvis, L. H. (1996). Young twoyear-olds' tendency to map novel verbs onto novel actions. Journal of Experimental Child Psychology, 63, 466-498.

Merriman, W. E., Marazita, J. \& Jarvis, L. (1995). Children's disposition to map new words onto new referents. In M. Tomasello \& W. E. Merriman (Eds.), Beyond names for things: Young children's acquisition of verbs (pp. 147-183). Hillsdale, NJ: Lawrence Erlbaum Associates.

Mather, E. \& Plunkett, K. (2012). The role of novelty in early word learning. Cognitive Science, $36(7), 1157-1177$.

Mayor, J. \& Plunkett, K. (2010). A neurocomputational account of taxonomic responding and fast mapping in early word learning. Psychological Review, 117(1), $1-31$.

Mervis, C. B. \& Bertrand, J. (1994). Acquisition of the novel name-nameless category (N3C) principle. Child Development, 65(6), 1646-1662.

Saffran, J. R., Aslin, R. N. \& Newport, E. L. (1996). Statistical learning by 8-month-old infants. Science, 274, 1926-1928.

Samuelson, L. K. (2002). Statistical regularities in vocabulary guide language acquisition in connectionist models and 15-20-month-olds. Developmental Psychology, 38, 10161037.

Samuelson, L. K. \& Smith, L. B. (1998). Memory and attention make smart word learning: An alternative account of Akhtar, Carpenter, and Tomasello. Child Development, 69, 94-104.

Simpson, A., Carroll, D. \& Riggs, K.J. (2014). Prepotency in action: Does children's knowledge of an artifact affect their ability to inhibit acting on it? Journal of Experimental Child Psychology, 118, 127-133. 
Smith, L. B., Jones, S., Landau, B., Gershkoff-Stowe, L. \& Samuelson, L. (2002). Object name learning provides on-the-job training for attention. Psychological Science, 13, 13 - 19.

Smith, L.B. \& Yu, C. (2008). Infants rapidly learn word-referent mappings via crosssituational statistics. Cognition, 106, 333-338.

Suanda, S. H. \& Namy, L. L. (2013). Young Word Learners' Interpretations of Words and Symbolic Gestures Within the Context of Ambiguous Reference. Child development, 84, 143-153

Swingley, D. (2010). Fast mapping and slow mapping in children's word learning. Language Learning and Development, 6, 179-183

Waxman, S. R. \& Booth, A. E. (2000). Principles that are invoked in the acquisition of words, but not facts. Cognition, 77, B33-B43.

Whiten, A., McGuigan, N., Marshall-Pescini, S. \& Hopper L. (2009). Emulation, imitation, overimitation and the scope of culture for child and chimpanzee. Philosophical Transactions of the Royal Society B, 364, 2117-2428.

Xu, J., Gannon, P., Emmorey, K., Smith, J.F. \& Braun, A. R. (2009). Symbolic gestures and spoken language are processed by a common neural system. Proceedings of the National Academy of Sciences, 106(49), 20664-20669. 
Figure 1: The familiar (top) and novel (bottom) objects used in Experiment 1 (Not to scale)

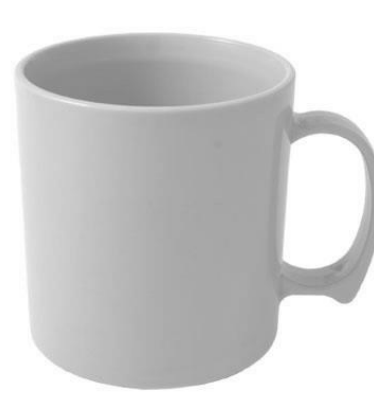

cup

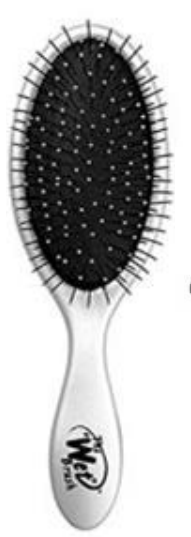

brush

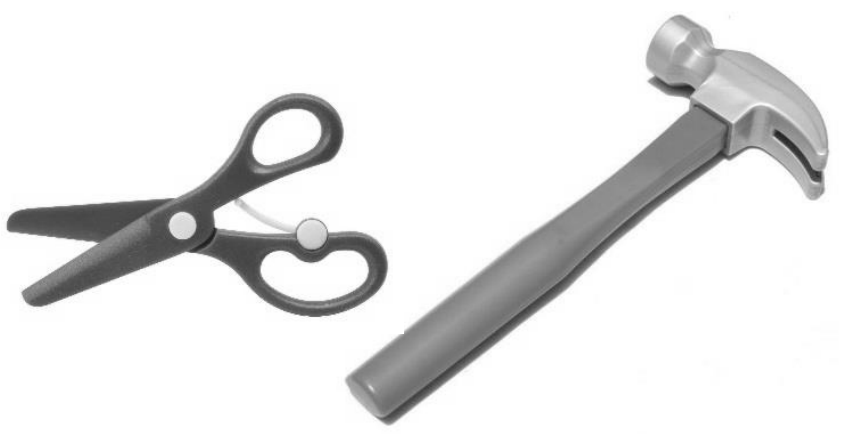

scissors hammer

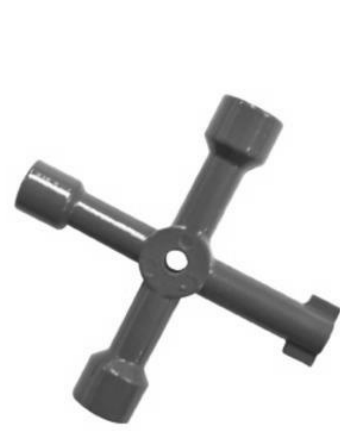

radiator key

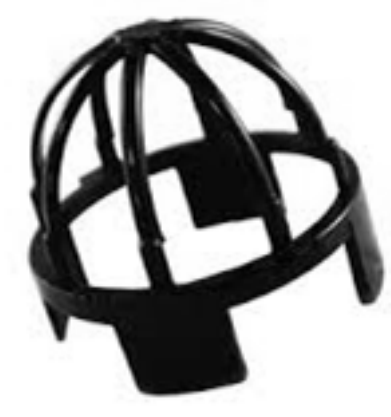

leaf guard

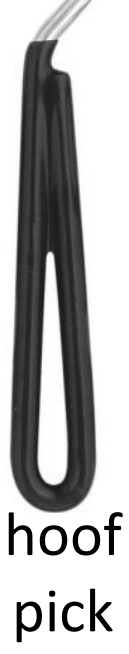

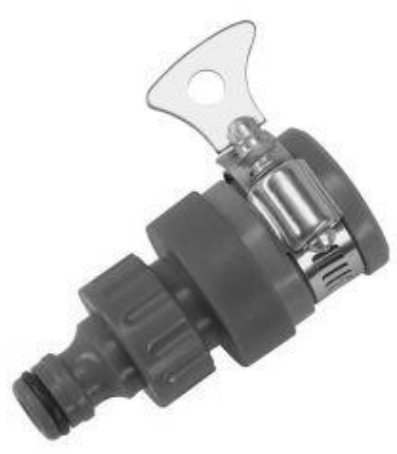

hose part 